\title{
Radiographic, biomechanical, and histological evaluation of rhBMP-2 in a 3-level intertransverse process spine fusion: an ovine study
}

\author{
Jeffrey M. Toth, PhD, ${ }^{1}$ Mei Wang, PhD, ${ }^{1}$ Joshua Lawson, MD, ${ }^{1}$ Jeffrey M. Badura, MS, ${ }^{2}$ and \\ Kimberly Bailey DuBose, $\mathrm{PhD}^{2}$
}

${ }^{1}$ Department of Orthopaedic Surgery, The Medical College of Wisconsin Inc., Milwaukee, Wisconsin; and 2Medtronic Spinal and Biologics, Memphis, Tennessee

\begin{abstract}
OBJECTIVE The objective of this study was to evaluate bone grafts consisting of rhBMP-2 on an absorbable collagen sponge with a ceramic composite bulking agent, rhBMP-2, directly on a ceramic-collagen sponge carrier or iliac crest bone graft (ICBG) in combination with local bone graft to effect fusion in a multisegmental instrumented ovine lumbar intertransverse process fusion model.
\end{abstract}

METHODS Thirty-six sheep had a single treatment at 3 spinal levels in both the right and left intertransverse process spaces. Group 1 sheep were treated with $7.5 \mathrm{~cm}^{3}$ of autograft consisting of ICBG plus local bone for each intertransverse process space. For Groups 2-4, $4 \mathrm{~cm}^{3}$ of local bone was placed within the intertransverse process space followed by $4.5-$ $5 \mathrm{~cm}^{3}$ of the rhBMP-2 graft material. Group 2 animals received $1.5 \mathrm{mg} / \mathrm{cm}^{3} \mathrm{rhBMP}-2$ on an absorbable collagen sponge with a commercial bone void filler consisting of Type I lyophilized collagen with a biphasic hydroxyapatite/ $\beta$-tricalcium phosphate ceramic with local bone. Group 3 animals received $0.75 \mathrm{mg} / \mathrm{m} \mathrm{cm}^{3}$ of rhBMP-2 on a collagen ceramic sponge carrier with local bone. Group 4 animals received $1.35 \mathrm{mg} / \mathrm{cm}^{3}$ of rhBMP-2 on the same collagen ceramic sponge carrier with local bone. Sheep were euthanized 6 months postoperatively. Manual palpation, biomechanical testing, CT, radiography, and undecalcified histology were performed to assess the presence of fusion associated with the treatments.

RESULTS All animals in Groups 2-4 that received grafts containing rhBMP-2 achieved radiographic and CT fusion at all 3 levels. In Group 1 (bone autograft alone), only 19\% of the levels demonstrated radiographic fusion, 14\% resulted in possible radiographic fusion, and $67 \%$ of the levels demonstrated radiographic nonfusion. Biomechanical testing showed that Groups 2-4 demonstrated similar stiffness of the L2-5 segment in all 6 loading directions, with each of the 3 groups having significantly greater stiffness than the autograft-only group. In Group 1, only 2 of 18 levels were rated as achieving bilateral histological fusion, with an additional 3 levels showing a unilateral fusion. The majority of the treated levels (13/18) in Group 1 were scored as histological nonfusions. There were no histological nonfusions in Groups 2 through 4. All 18 levels in Group 2 were rated as bilateral histological fusions. A majority (34/36) of the levels in Group 3 were rated as bilateral histological fusions, with 2 levels showing a unilateral fusion. A majority (35/36) of the levels in Group 4 were rated as bilateral histological fusions, with 1 level showing a unilateral fusion.

CONCLUSIONS In the ovine multilevel instrumented intertransverse process fusion model, rhBMP-2 was able to consistently achieve CT, radiographic, biomechanical, and histological fusion. Compared with ICBG, the gold standard for bone grafting, rhBMP-2 was statistically superior at achieving radiographic and histological fusion.

http://thejns.org/doi/abs/10.3171/2016.4.SPINE151316

KEY WORDS rhBMP-2; multilevel spine fusion; lumbar fusion; autograft

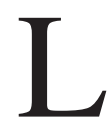
UMBAR spine fusion is a commonly performed surgical procedure for patients with spinal instability and/or other spinal pathologies, often with concomitant back pain. It is estimated that more than 400,000 spinal arthrodeses were performed in the United States per year in 2008. ${ }^{13}$ Most spinal arthrodesis procedures consist of a bone graft and/or a bone graft substitute in conjunction with posterior instrumentation consisting of pedicle screws and rods. Although autograft is frequently used as the bone graft for spinal fusion, it has several limitations, including the quantity and quality of the bone graft that can be harvested, as well as donor site pain, infection, and do-

ABBREVIATIONS ACS = absorbable collagen sponge; $C C S=$ collagen ceramic sponge; $H A=$ hydroxyapatite; ICBG $=$ iliac crest bone graft; OLR = ordinal logistic regression; $\mathrm{PA}=$ posteroanterior; rhBMP-2 = recombinant human bone morphogenetic protein-2; TCP-HA = tricalcium phosphate .

SUBMITTED November 18, 2015. ACCEPTED April 26, 2016.

INCLUDE WHEN CITING Published online July 1, 2016; DOI: 10.3171/2016.4.SPINE151316. 
nor site morbidity. In addition, the efficacy of bone graft to effect arthrodesis decreases as more levels are attempted.

Clinically, multiple-level fusions are performed for various spinal conditions, especially for scoliosis and correcting spinal deformities, although the failure of bone autograft to induce spinal fusion may occur in as many as $25 \%$ to $33 \%$ of the attempted multilevel fusions..$^{1,2,4,6} \mathrm{~A}$ few studies have investigated the use of osteoinductive bone graft substitutes in multilevel fusions to reduce pseudarthrosis rates associated with bone autograft.,10,12 Mulconrey et al. conducted a prospective radiographic analysis of multilevel fusions for posterior adult spinal deformity performed with recombinant human bone morphogenetic protein-2 (rhBMP-2) in a group of patients with medical comorbidities, tobacco use, preoperative pseudarthrosis, and previous revision surgery. ${ }^{12}$ In 43 of these patients, they found a $97 \%$ fusion rate with $20 \mathrm{mg} / \mathrm{level}$ of rhBMP-2 on absorbable collagen sponge (ACS) with local bone graft and a bulking agent (tricalcium phosphate/hydroxyapatite [TCP-HA]) for multilevel posterolateral fusions (156 levels, with an average of 3.63 levels/patient). ${ }^{12}$ In an additional 8 patients treated with $40 \mathrm{mg}$ per level of rhBMP-2 on ACS with TCP-HA with no autologous bone, they found a $100 \%$ fusion rate for multilevel posterolateral fusions (43 levels, with an average of 5.38 levels/patient). ${ }^{12}$ The authors concluded that in multilevel spinal fusion, rhBMP-2 eliminated the necessity for iliac crest bone graft (ICBG) and yielded an excellent fusion rate. Similarly, Luhmann et al. found a 93\% fusion rate for multilevel posterior fusion in 41 patients with the use of a mean of 13.7 mg of rhBMP-2 per level. ${ }^{10}$

The preclinical effectiveness of bone grafts and bone graft substitutes are most commonly examined in singlelevel animal models. However, as more fusion levels are attempted-in animals as well as humans-the efficacy of the treatment in generating a spinal fusion decreases. For this reason, assuming a similar effectiveness of bone grafts or graft substitutes for multiple-level fusions based on efficacy in single-level models is a logical error and can lead to pseudarthrosis in a multilevel fusion.

Only a few authors have studied bone graft or graft substitutes in multilevel animal models. Mermer et al. published results in a challenging multilevel uninstrumented sheep model. ${ }^{11}$ The authors concluded, "Neither autograft nor OP-1 [rhBMP-7] could fuse all three levels in any specimen." 11 Mermer et al. found a high rate of nonunion with the ovine multilevel uninstrumented fusion model using autograft or rhBMP-7. Steffen et al. evaluated coralline calcium phosphate granules, ICBG, and a $50 / 50$ mix of coralline calcium phosphate with autograft in a 2-level instrumented spine study in skeletally mature Dorset sheep at 20 weeks. ${ }^{14}$ In their study, the coralline granules mixed with autograft had fusion outcomes similar to bone autograft. ${ }^{14}$ However, large neutral zone measurements detected by biomechanical testing suggested segmental laxity, interpreted as nonunions by the authors. ${ }^{14}$ Kim et al. used an L2-3 and L5-6 noncontiguous multilevel instrumented fusion model to evaluate iliac crest bone autograft as well as Healos MP52 in the ovine model at 6 and 12 months. ${ }^{8}$ The authors found comparable bone formation by backscatter electron measurements and stated, "Overall HealosMP52 stimulated the formation of fusion masses that were histologically comparable to autograft-treated fusion sites." 8

The objective of this study was to evaluate bone grafts consisting of rhBMP-2 on ACS with a ceramic composite bulking agent, rhBMP-2, directly on a collagen ceramic sponge (CCS) carrier, or ICBG in combination with local bone graft to effect lumbar intertransverse process fusion at 6 months postoperatively in a multisegmental instrumented ovine lumbar intertransverse process fusion model. The bone graft and graft substitutes were evaluated by manual palpation, CT, high-resolution radiography, biomechanical testing, and undecalcified histology with microradiography.

\section{Methods}

\section{Animal Groups and Surgical Procedure}

The sheep lumbar spine model was chosen because of the biomechanical similarities between lumbar spines in sheep and humans. ${ }^{3}$ The study was performed in accordance with the Guide for the Care and Use of Laboratory Animals (Institute for Laboratory Animal Research, National Research Council. Washington, DC: National Academy Press, 1996). Under an Institutional Animal Care and Use Committee-approved protocol, each of the 36 sheep had a single treatment at all 3 spinal levels (both the right and left intertransverse process spaces). The intended spinal levels were L2-3, L3-4, and L4-5. Treatments are shown in Table 1. Group 1 sheep were treated with $7.5 \mathrm{~cm}^{3}$ of autograft consisting of ICBG plus local bone in each intertransverse process space. For Groups $2-4,4 \mathrm{~cm}^{3}$ of local bone was placed within the intertransverse process space followed by 4.5 to $5 \mathrm{~cm}^{3}$ of the rhBMP-2 graft material. Animals in Group 2 received $1.5 \mathrm{mg} / \mathrm{cm}^{3} \mathrm{rhBMP}-2$ on an ACS with a commercial bone void filler consisting of Type I lyophilized collagen with a biphasic (15\% hydroxyapatite $[\mathrm{HA}]$ and $85 \% \beta$-tricalcium phosphate $[\mathrm{TCP}]$ ) ceramic (MASTERGRAFT Strip, Medtronic) with local bone (http://www.accessdata.fda.gov/cdrh_docs/pdf8/K082166. pdf). The ceramic-to-collagen ratio was $96.5 \%$ (wt $\%$ ) biphasic HA/ $\beta$-TCP ceramic to $3.5 \%$ (wt\%) collagen. Animals in Group 3 received $0.75 \mathrm{mg} / \mathrm{cm}^{3}$ of rhBMP-2 on a CCS carrier with local bone. The ceramic-to-collagen ratio of the CCS material was $83 \%$ (wt\%) biphasic HA/ $/$-TCP ceramic to $17 \%$ (wt\%) collagen. Group 4 consisted of 1.35 $\mathrm{mg} / \mathrm{cm}^{3}$ of rhBMP-2 on the same CCS carrier with local bone. At 6 months postoperatively, the sheep were euthanized, and the lumbar spine was removed for evaluation.

\section{Manual Palpation}

The treated segments (L2-5 was intended) were dissected from the harvested lumbar spine and cleaned of extraneous soft tissues, leaving the ligamentous and osseous tissues intact. After removal of fusion instrumentation, manual palpation of the treated spinal levels was performed. Similar to the methods used in other studies, each spinal level was evaluated by 2 evaluators blinded to treatment based on intersegmental movement in 3 anatomical planes. ${ }^{7,9,11}$ The spinal level was graded a solid fusion if there was no detectable motion in any plane, or it 
TABLE 1. Treatment and group assignment

\begin{tabular}{ccc}
\hline Group No. & \multicolumn{1}{c}{ Treatment } & No. of Sheep \\
\hline 1 & ICBG + LB & 6 \\
\hline 2 & $\begin{array}{c}1.5 \mathrm{mg} / \mathrm{ml} \text { rhBMP-2/ACS + } \\
\text { MASTERGRAFT Strip + LB }\end{array}$ & 6 \\
& $0.75 \mathrm{mg} / \mathrm{ml} \mathrm{rhBMP-2/CCS} \mathrm{+} \mathrm{LB}$ & 12 \\
\hline 3 & $1.35 \mathrm{mg} / \mathrm{ml} \mathrm{rhBMP-2/CCS} \mathrm{+} \mathrm{LB}$ & 12 \\
\hline 4
\end{tabular}

$\mathrm{LB}=$ local bone.

was graded pseudarthrosis if any degree of motion was observed.

\section{Ex Vivo Biomechanical Testing of the Treated Lumbar Motion Segments}

Unconstrained biomechanical testing was performed in a nondestructive manner on the treated multilevel spinal fusion segments. Flexibility of the motion segments was determined in flexion, extension, right and left lateral bending, and right and left axial rotation. The most inferior vertebra of the 3-level fusion segment was potted in dental cement on the base frame. Moments were applied to the most superior vertebra of the fusion segment through a specially designed loading frame.

Static loads were used to apply the pure moments in 6 steps of $0,0.5,1.5,2.5,4.5$, and $6.5 \mathrm{Nm}$. A 6-axis load cell was placed in series with all tested specimens to verify the applied moments. 3D rotation at each of the 3 fused motion segments and between the most superior and the most inferior vertebrae was measured simultaneously with a motion analysis system (Optotrak Certus Motion Tracking system, Northern Digital Inc.). Three active markers were attached to each vertebra, from L-2 to L-5. Rotational flexibility (stiffness) was derived from moment-angular displacement plots using linear regression for the entire fusion segment (L2-5). Biomechanics data from a normal (untreated) group of sheep with the L2-3 and L4-5 motion segments intact have been obtained previously and were used as baseline data for normalization of treated groups. ${ }^{3}$

\section{Radiography}

A high-resolution radiography unit (Faxitron model 43805, Hewlett Packard) and high-resolution film (EKTASCAN B/RA Film 4153) were used to produce a high-resolution posteroanterior (PA) radiograph of the treated lumbar spinal levels. Two blinded scorers evaluated the resulting Faxitron radiographs for intertransverse process fusion. On both the right and left sides of each level on the PA radiograph, the intertransverse process space was evaluated for fusion based on the following scoring method: 4 , continuous bony bridging; 3 , increased bone density throughout the intertransverse process space but lucency, which interrupts continuous bony bridging; 2 , mostly lucencies with some nonbridging bone in the intertransverse process space; and 1, nonfusion with no bone in the intertransverse process space. Based on both the right and left sides of the PA radiographs, the blinded evaluators rated an overall fusion score for each spinal level using the following criteria: 3 , solid fusion: solid intertransverse process fu- sion on right and left with no radiolucencies that interrupt continuous superior to inferior bony bridging; 2 , possible fusion: intertransverse process fusion on the right or left, but not both (lucencies in the intertransverse process space on right or left that could interrupt continuous superior to inferior bony bridging); 1 , nonfusion: isolated bone formation without continuous superior to inferior bony bridging on both right and left sides (significant lucency with no evidence of continuous superior to inferior bony bridging [intertransverse process fusion] on the right or left).

\section{Computed Tomography}

CT imaging was performed using parameters appropriate for bone at a 1-mm slice thickness. Sagittal and coronal plane reconstructions were obtained for all treated sheep. CT scans were evaluated by 2 independent veterinary surgeon reviewers. Any levels with one assessment of "fused" and the other of "not fused" were adjudicated by a third reviewer, and that score was used. For an animal to be considered to have achieved fusion on CT, all levels on both right and left sides had to be fused.

\section{Undecalcified Histology and Microradiography}

Immediately after biomechanical testing, the treated spinal levels were dissected into individual functional spinal units and further sectioned into right lateral, right medial, left lateral, and left medial blocks for histology and immersion fixed in 70\% ethanol. Using a band saw, the identified right and left L-3 transverse processes were bisected so that half of each L-3 transverse process remained with the L2-3 fusion mass and the other half remained with the L3-4 fusion mass. The same technique was repeated so that the left L-4 transverse process was bisected so that half of each L-4 transverse process remained with the L3-4 fusion mass and the other half remained with the L4-5 fusion mass. All tissue samples were labeled, fixed in ethanol, sequentially dehydrated in alcohols, cleared in xylene, and embedded in graded catalyzed methyl methacrylate. Sectioning and differential staining along with qualitative optical microscopy were performed to assess bone bridging and extent of fusion associated with the bone graft and bone graft substitutes. Selected undecalcified sections from the treated lumbar spinal levels were radiographed using a microradiography unit and spectroscopic film. Sections were placed on the high-resolution film and exposed to the x-ray source at $20 \mathrm{kV}$ and $3 \mathrm{~mA}$ for approximately 45 seconds for each $100 \mu \mathrm{m}$ of section thickness.

\section{Histological Section Fusion Criteria}

For each individual histological section, a score of 2 was recorded if a continuous bony bridge (histological fusion) was observed in that section. A score of 1 was recorded if a histological nonfusion with an incomplete bony bridge with de novo bone was found in $>50 \%$ of the length of the section. A score of 0 was recorded if a histological nonfusion with an incomplete bony bridge with de novo bone was found in $<50 \%$ of the length of the section.

\section{Histological Level Fusion Criteria}

For each treated level, a bilateral fusion existed if con- 
TABLE 2. Manual palpation fusion results

\begin{tabular}{cc}
\hline Group No. & Incidence of Fusion by Manual Palpation \\
\hline 1 & $4 / 6$ \\
\hline 2 & $6 / 6$ \\
\hline 3 & $12 / 12$ \\
\hline 4 & $12 / 12$ \\
\hline
\end{tabular}

tinuous bony bridging was found in $\geq 50 \%$ of the sections for the level on the right and the left. A unilateral fusion existed if continuous bony bridging was found in $\geq 50 \%$ of the sections for the level on the right or the left. A histological nonfusion existed if continuous bony bridging was found in $<50 \%$ of the sections for the level on the right and the left. For each treated level, the presence/absence of residual ceramic was also graded.

\section{Statistical Analysis}

Contingency table and chi-square tests were performed to evaluate the differences between manual palpation results for the 4 treatment groups. Radiographic and histological data were statistically analyzed using ordinal logistic regression. The kappa coefficient and the Kendall's tau coefficient for measures of association were used to evaluate CT interrater reliability. Differences in the segmental stiffness (flexibility) between the fusion treatments and the normal intact spines were statistically compared. The Shapiro-Wilk test was used to test for normality of the biomechanics data. Based on a normality check, parametric ANOVA or nonparametric tests, such as the Wilcoxon test, were used to analyze the biomechanics data. Ordinal logistic regression (OLR) analysis was used to compare histological fusion results between treatment groups. All statistical analyses were conducted using commercial statistical software (JMP version 11, SAS Institute Inc.).

\section{Results \\ Manual Palpation}

The incidence of fusion by manual palpation for all treatment groups is presented in Table 2. Manual palpation results showed equivalence among the rhBMP-2 groups; all animals from Groups 2 through 4 demonstrated no movement or very limited movement at the 3 fusion levels, which was graded as solid fusion. In Group 1, gross movement was detected at all 3 levels in 2 of the 6 animals and was graded as pseudarthrosis, while in the remaining 4 the outcome was graded as solid fusion.

\section{Radiography}

Representative high-resolution PA radiographs from Groups 1 through 4 are shown in Fig. 1. Radiographic results showed an equivalence among the rhBMP-2 groups, as all animals from Groups 2 through 4 achieved radiographic fusion at all 3 levels. In Group 1 (bone autograft alone), only $19 \%$ of the levels demonstrated radiographic fusion, $14 \%$ resulted in possible radiographic fusion, while $67 \%$ of the levels demonstrated radiographic nonfusion. Nonparametric comparisons using the Dunn method with Group 1 as the control demonstrated that the difference between Group 1 and the other 3 groups in terms of radiographic fusion was statistically significant $(\mathrm{p}<0.0001)$.

\section{Computed Tomography}

CT fusion data are presented in Table 3. In the autograft group, only 1 sheep exhibited evidence that all 3 levels had fused. One sheep from Group 1 had 2 of 3 levels fused and 1 additional sheep had 2 sides fused. All animals from Groups 2 through 4 containing rhBMP-2 achieved fusion at all 3 levels as assessed by CT. The interrater agreement statistic between Evaluator 1 and Evaluator 2 for the 6-month CT scores was calculated. The kappa coefficient was 0.65 (95\% CI $0.55-0.76, \mathrm{p}<0.0001)$, representing
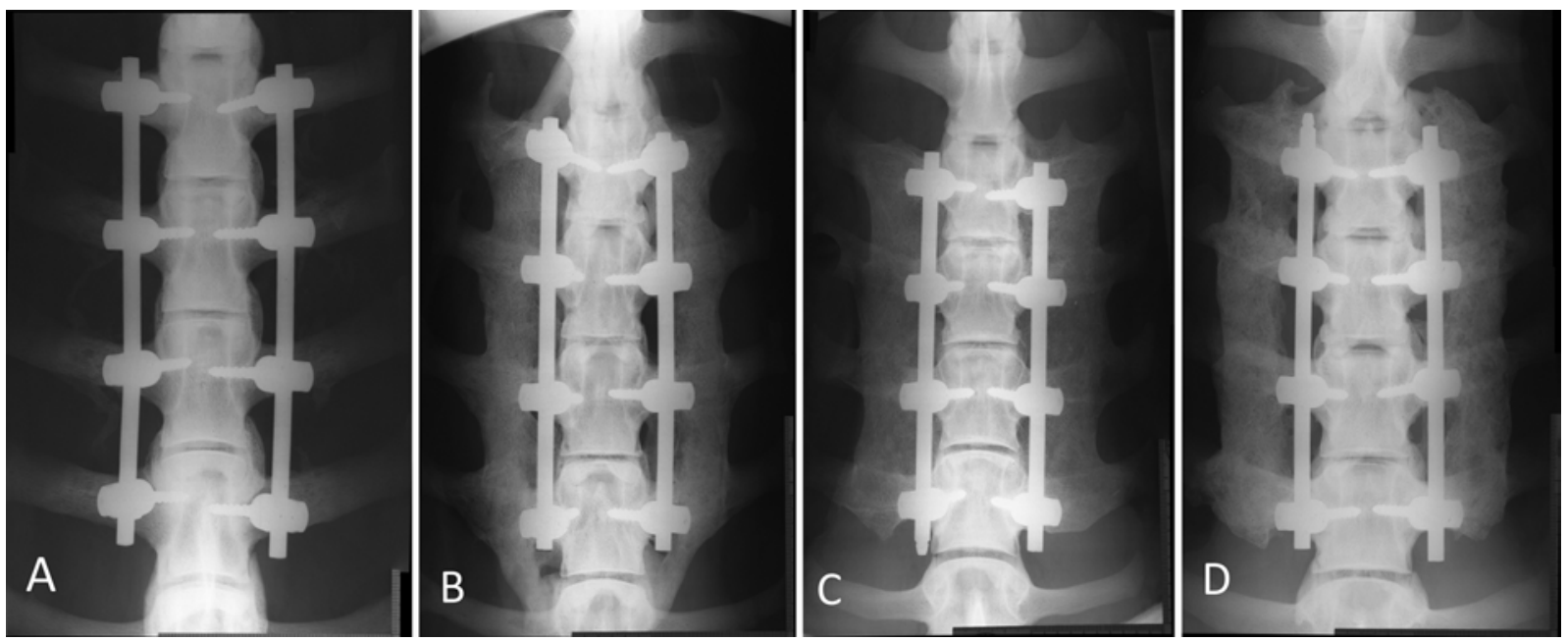

FIG. 1. Representative high-resolution PA radiographs obtained in 1 sheep from each group. A: Image showing bilateral nonfusion from a Group 1 treated sheep. A millimeter scale is seen at the lower right. B: Image showing bilateral multilevel fusion from a Group 2 treated sheep. A millimeter scale is seen at the lower right. C: Image showing bilateral multilevel fusion from a Group 3 treated sheep. A millimeter scale is seen at the lower right. D: Image showing bilateral multilevel fusion from a Group 4 treated sheep. A millimeter scale is seen at the lower right. 
TABLE 3. CT fusion results

\begin{tabular}{cc}
\hline Group No. & Incidence of CT Fusion \\
\hline 1 & $1 / 6$ \\
\hline 2 & $6 / 6$ \\
\hline 3 & $12 / 12$ \\
\hline 4 & $11 / 11$ \\
\hline
\end{tabular}

substantial agreement between the 2 evaluators. In addition, for measures of association, the Kendall's tau coefficient was 0.85 (95\% CI 0.78-0.93, p < 0.0001).

\section{Biomechanical Testing}

The Shapiro-Wilk test rejected the normal distribution hypothesis in a majority of the biomechanical testing trials. Therefore, nonparametric Wilcoxon multiple paired comparisons were conducted. The median stiffness $(25 \%$ and $75 \%$ quartiles) of the 3-level L2-5 fusion segment is presented in Fig. 2. When comparing the stiffness of the L2-5 segment among the 4 treatment groups, Groups 2-4 demonstrated similar stiffness in all 6 loading directions, with each of the 3 groups having significantly greater stiffness than Group 1. The median stiffness of Group 1 was 5.5 and $5.2 \mathrm{Nm} /{ }^{\circ}$ in the right and left axial rotations, respectively; 4.8 and $4.1 \mathrm{Nm} /{ }^{\circ}$ in the right and left lateral bending, respectively; and 2.8 and $1.8 \mathrm{Nm} /{ }^{\circ}$ in flexion and extension, respectively. The median stiffness of the remaining 3 treatment groups was 2.4 to 3.0 times stiffer than Group 1 in right axial rotation (Kruskal-Wallis, $\mathrm{p}<$ $0.005), 3.0$ to 3.3 times stiffer in left axial rotation ( $\mathrm{p}<$ 0.04 ), 11.9 to 13.2 times stiffer in right lateral bending ( $\mathrm{p}$ $<0.006), 12.0$ to 16.0 times stiffer in left lateral bending $(\mathrm{p}<0.003), 3.8$ to 3.9 times stiffer in flexion $(\mathrm{p}<0.008)$, and 4.4 to 4.9 times stiffer in extension $(\mathrm{p}<0.01)$. Good correlation was found between biomechanical flexibility test results and Faxitron radiographic fusion scores. All 4 treatment groups demonstrated significantly higher stiffness and lower ranges of motion than the historical intact control. ${ }^{3}$ The increases in stiffness over the intact spines were most pronounced in the loading direction of lateral bending.

\section{Histology}

In Group 1 (ICBG + local bone group), only 2 of 18 levels were rated as bilateral histological fusion, with an additional 3 levels showing a unilateral fusion. The majority of the treated levels (13/18) in Group 1 were scored as histological nonfusions. A representative composite microradiograph showing a histological nonfusion in Group 1 is seen in Fig. 3A. There were no histological nonfusions in Groups 2 through 4. Representative composite microradiographs showing histological fusions in Groups 2 through 4 are seen in Fig. 3B, C, and D, respectively. All 18 levels in Group 2 were rated as bilateral histological fusions. A majority (34/36) of the levels in Group 3 were rated as bilateral histological fusions, with 2 levels showing a unilateral fusion. A majority (35/36) of the levels in Group 4 were rated as bilateral histological fusions, with 1 level showing a unilateral fusion. Histological fusions in the rhBMP-2-treated sheep correlated well with CT fusion results and radiographic fusion assessments, as well as biomechanical testing data. The 3 rhBMP-2 treatment groups (Groups 2 through 4) had a higher odds of achieving a higher fusion score than the ICBG group (Group 1) based on ordinal logistic regression analysis $(\mathrm{p}<0.0001)$. There was no significant difference between Groups 2,3 , and $4(\mathrm{p}<0.43)$.

As seen in Fig. 3B-D, incorporated residual ceramic was observed in the 3 rhBMP-2 treatment groups. There was no significant difference in the amount of residual ceramic between Groups 2 through 4 (OLR analysis, $p$ $<0.33$ ). Local bone was difficult to differentiate from de novo bone of the fusion mass. When observed, local bone appeared to be incorporated into the ventral aspect of the dorsal fusion masses. This would be consistent with the ventral placement of the local bone in the intertransverse space prior to placement of the rhBMP-2 graft material.

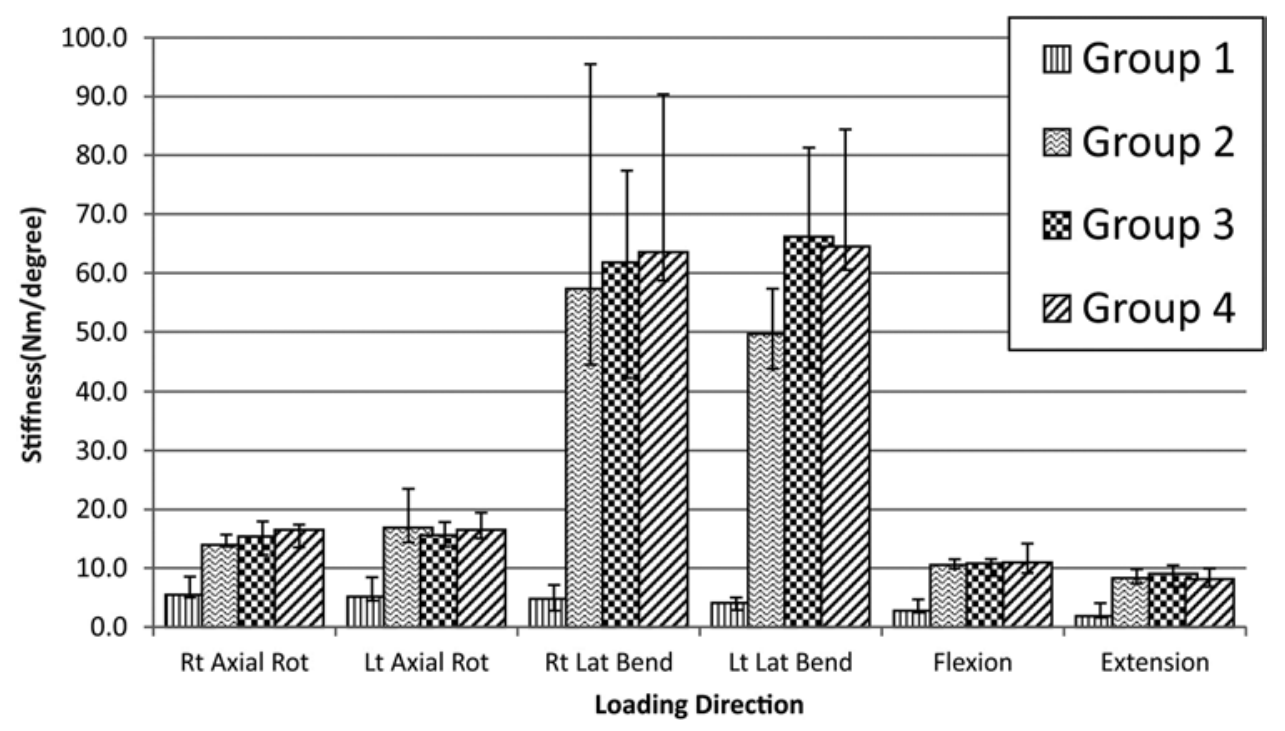

FIG. 2. Biomechanical median stiffness in $\mathrm{Nm} /{ }^{\circ}(25 \%$ and $75 \%$ quartiles) of the 3 -level fusion segments for all 4 treatment groups. 

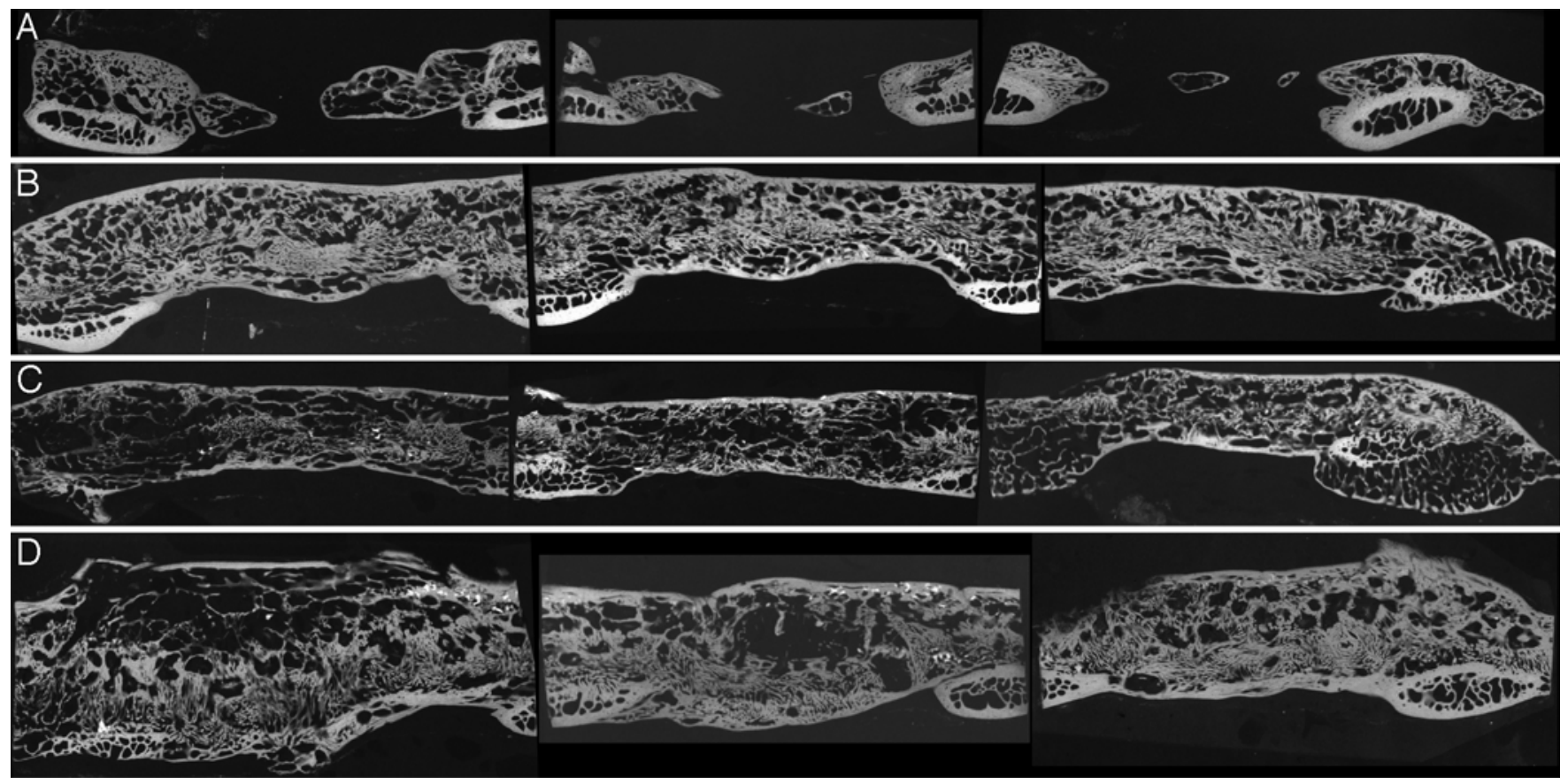

FIG. 3. Composite microradiographs. A: Undecalcified section showing a nonunion with incomplete bony bridging in the 3 intertransverse process spaces dorsal to the decorticated transverse processes in a Group 1 treated sheep. B: Undecalcified section showing solid fusion masses at 3 levels dorsal to the decorticated transverse processes with incorporated residual radiopaque ceramic granules in a Group 2 treated sheep. C: Undecalcified section showing solid fusion masses at 3 levels dorsal to the decorticated transverse processes with few incorporated residual radiopaque ceramic granules in a Group 3 treated sheep. D: Undecalcified section showing solid fusion masses at 3 levels dorsal to the decorticated transverse processes with few incorporated residual radiopaque ceramic granules in a Group 4 treated sheep. An unusual circular fine cancellous bony architecture is seen in this microradiograph. Original magnification $\times 3.5$.

As seen in Fig. 3D, a circular fine cancellous bony architecture was occasionally observed within the fusion masses in the 3 rhBMP-2 treatment groups. This unusual fine cancellous architecture was found in $22 \%$ of the treated levels in Group 2, 31\% of the treated levels in Group 3, and in $33 \%$ of the treated levels in Group 4. In addition, blood accumulation was found in $11 \%$ of the treated levels in Group 2,33\% of the treated levels in Group 3, and in $28 \%$ of the treated levels in Group 4.

\section{Discussion}

In the multilevel instrumented sheep intertransverse process fusion model, rhBMP-2 consistently achieved radiographic, biomechanical, and histological fusion. CT fusion results, radiograph fusion results, manual palpation results, and histology showed equivalence among the 3 rhBMP-2 treatment groups. Compared with ICBG, the gold standard for bone grafting, rhBMP-2 was statistically superior at achieving radiographic and histological fusion. The relatively low fusion rates for ICBG are consistent with the previous literature and demonstrate that the 3-level sheep instrumented intertransverse process fusion model is indeed a challenging model. ${ }^{11}$ If the bone graft substitutes have a similar clinical efficacy, the rhBMP-2 bone graft substitutes could obviate the morbidity and pain associated with obtaining sufficient graft volume to effect multilevel spine fusions in humans.

Similar to the manual palpation method used in other studies reported in the literature, each spinal level was evaluated based on spine movement in 3 anatomical planes by 2 evaluators blinded to treatment. ${ }^{7,9,11}$ Although manual palpation has been used in previous studies to detect fusion, in the current study, manual palpation was not a sensitive method to detect pseudarthroses that were detectable by radiography, formal biomechanics, and histology. Specifically, by histology, there were 13 nonfused levels in Group 1 (ICBG + local bone group). Similarly, at least 1 radiograph reviewer assigned a score of 1 (radiographic nonfusion) to 12 of the iliac crest bone autograft (Group 1) levels in this study. Manual palpation was only capable of detecting 2 sheep in Group 1, which were graded as pseudarthroses. Thus, at least in the multilevel sheep model, manual palpation was not sensitive to pseudarthroses.

There was no evidence of unusual cancellous bony architecture found in Group 1 (ICBG + local bone group). An unusual circular fine cancellous bony architecture, sometimes surrounding dense calcifications, was noted in the rhBMP-2 treatment groups. Zara et al. have previously reported cyst-like bone formation with high doses of rhBMP-2 in a rat femoral segmental defect model..$^{15}$ Their work shows that lower doses of rhBMP-2 did not induce cyst-like bone formation that they described as "abnormal" bone formation. In the current study, the circular fine cancellous bony architecture was not abnormal and was not more prevalent in the high-dose rhBMP-2 group (Group 4) than in the low-dose rhBMP-2 group (Group 3). 
In addition, the bony architecture noted in the histology in our study did not affect radiographic fusion assessment or biomechanical stiffness results.

\section{Conclusions}

In the multilevel instrumented sheep intertransverse process fusion model, rhBMP-2 was able to consistently achieve radiographic, biomechanical, and histological fusion. Compared with ICBG, the gold standard for bone grafting, rhBMP-2 was statistically superior at achieving radiographic and histological fusion. Even with pedicle screw and rod instrumentation, the relatively low fusion rates for ICBG demonstrate that the 3-level sheep instrumented intertransverse process fusion model is indeed a challenging model. The rhBMP-2 bone graft substitutes could obviate the morbidity and pain associated with obtaining sufficient graft volume to effect multilevel spine fusions. The 3-level intertransverse process fusion animal model has been used to compare bone graft materials. Similar to published clinical and animal studies, the current study confirmed the challenging nature of achieving multisegment intertransverse process fusion while demonstrating the potential for increased fusion rates with rhBMP-2.

\section{Device Status/Drug Statement}

The device(s)/drug(s) that is/are the subject of this manuscript is/are exempt from FDA or corresponding national regulations because this is an experimental study conducted in sheep. This is not a human clinical study.

\section{Acknowledgments}

We thank and acknowledge funding from Medtronic Spinal and Biologics for research support of the study. We thank and acknowledge Linnea Lentz, DVM, PhD, Noah Barka, DVM, and Douglas Fredericks for their expertise in performing the surgical procedures. We thank and acknowledge Amy Rizzo, MT (ASCP), for technical expertise in histologic processing of the tissues as well as Linda McGrady, MS, for technical expertise in biomechanical testing.

\section{References}

1. Cleveland M, Bosworth DM, Thompson FR: Pseudarthrosis in the lumbosacral spine. J Bone Joint Surg Am 30A:302312,1948

2. Deguchi M, Rapoff AJ, Zdeblick TA: Posterolateral fusion for isthmic spondylolisthesis in adults: analysis of fusion rate and clinical results. J Spinal Disord 11:459-464, 1998

3. Easley NE, Wang M, McGrady LM, Toth JM: Biomechanical and radiographic evaluation of an ovine model for the human lumbar spine. Proc Inst Mech Eng H 222:915-922, 2008

4. Flatley TJ, Derderian H: Closed loop instrumentation of the lumbar spine. Clin Orthop Relat Res (196):273-278, 1985

5. Glassman SD, Carreon L, Djurasovic M, Campbell MJ, Puno RM, Johnson JR, et al: Posterolateral lumbar spine fusion with INFUSE bone graft. Spine J 7:44-49, 2007

6. Herkowitz HN, Sidhu KS: Lumbar spine fusion in the treatment of degenerative conditions: current indications and recommendations. J Am Acad Orthop Surg 3:123-135, 1995

7. Kanayama M, Cunningham BW, Sefter JC, Goldstein JA,
Stewart G, Kaneda K, et al: Does spinal instrumentation influence the healing process of posterolateral spinal fusion? An in vivo animal model. Spine (Phila Pa 1976) 24:10581065,1999

8. Kim DH, Jahng TA, Fu TS, Zhang HY, Novak SA: Evaluation of HealosMP52 osteoinductive bone graft for instrumented lumbar intertransverse process fusion in sheep. Spine (Phila Pa 1976) 29:2800-2808, 2004

9. Louis-Ugbo J, Murakami H, Kim HS, Minamide A, Boden SD: Evidence of osteoinduction by Grafton demineralized bone matrix in nonhuman primate spinal fusion. Spine (Phila Pa 1976) 29:360-366, Z1, 2004

10. Luhmann SJ, Bridwell KH, Cheng I, Imamura T, Lenke LG, Schootman M: Use of bone morphogenetic protein-2 for adult spinal deformity. Spine (Phila Pa 1976) 30 (17 Suppl):S110-S117, 2005

11. Mermer MJ, Gupta MC, Wheeler DL, Helgerson J, Reddi AH, Hazelwood S, et al: Efficacy of osteogenic protein-1 in a challenging multilevel fusion model. Spine (Phila Pa 1976) 29:249-256, 2004

12. Mulconrey DS, Bridwell KH, Flynn J, Cronen GA, Rose PS: Bone morphogenetic protein (RhBMP-2) as a substitute for iliac crest bone graft in multilevel adult spinal deformity surgery: minimum two-year evaluation of fusion. Spine (Phila Pa 1976) 33:2153-2159, 2008

13. Rajaee SS, Bae HW, Kanim LE, Delamarter RB: Spinal fusion in the United States: analysis of trends from 1998 to 2008. Spine (Phila Pa 1976) 37:67-76, 2012

14. Steffen T, Marchesi D, Aebi M: Posterolateral and anterior interbody spinal fusion models in the sheep. Clin Orthop Relat Res (371):28-37, 2000

15. Zara JN, Siu RK, Zhang X, Shen J, Ngo R, Lee M, et al: High doses of bone morphogenetic protein 2 induce structurally abnormal bone and inflammation in vivo. Tissue Eng Part A 17:1389-1399, 2011

\section{Disclosures}

The authors report the following. Dr. Toth: consult for and received clinical or research support for the study described from Medtronic. Dr. Lawson: stock ownership in Stryker. Mr. Badura: employee of, direct stock ownership in, and received clinical or research support for the study described from Medtronic. Dr. DuBose: employee of Medtronic. Institutional research support was received from Medtronic in support of this research.

\section{Author Contributions}

Conception and design: Badura. Acquisition of data: Toth, Wang. Analysis and interpretation of data: Toth, Wang. Drafting the article: Toth, Badura. Critically revising the article: Toth, Badura. Reviewed submitted version of manuscript: all authors. Approved the final version of the manuscript on behalf of all authors: Toth. Statistical analysis: Wang. Study supervision: Toth, Badura, Bailey DuBose.

\section{Supplemental Information}

\section{Previous Presentations}

Portions of this work were presented in abstract form as proceedings at the 2016 Annual Meeting of the Orthopaedic Research Society, Orlando, Florida, March 5-8, 2016.

\section{Correspondence}

Jeffrey M. Toth, Department Orthopaedic Surgery, The Medical College of Wisconsin, Inc., 9200 W Wisconsin Ave., Box 26099, Milwaukee, WI 53226-0099. email: jtoth@mcw.edu. 